\title{
Belimumab use, clinical outcomes and glucocorticoid reduction in patients with systemic lupus erythematosus receiving belimumab in clinical practice settings: results from the OBSErve Canada Study
}

\author{
Zahi Touma $^{1} \cdot$ Amyn Sayani $^{2} \cdot$ Christian A. Pineau $^{3} \cdot$ Isabelle Fortin $^{4} \cdot$ Mark Matsos $^{5} \cdot$ \\ George A. Ecker ${ }^{6,7} \cdot$ Andrew Chow $^{8} \cdot$ Sandra Iczkovitz $^{2}$
}

Received: 23 November 2016 / Accepted: 16 February 2017 / Published online: 9 March 2017

(c) The Author(s) 2017. This article is published with open access at Springerlink.com

\begin{abstract}
To describe the characteristics of patients receiving belimumab, overall patterns of systemic lupus erythematosus (SLE) care, clinical outcomes, and changes in glucocorticoid dose following 6 months of therapy with belimumab, and healthcare resource utilization in belimumab users in Canadian clinical practice settings. Retrospective multicenter medical chart review study of adult patients with SLE who were prescribed belimumab as part of usual care and who received $\geq 8$ infusions or 6 months of treatment. Primary endpoints included physiciandetermined overall clinical improvement from baseline, glucocorticoid use, and physician-determined SLE disease severity at Month 6. In total, 52 patients were included in the study. At belimumab initiation, 5.8/76.9/17.3\% of patients had mild/moderate/severe SLE, respectively. Oral glucocorticoids were discontinued in $11.4 \%$ of patients
\end{abstract}

Zahi Touma

zahi.touma@uhn.ca

Sandra Iczkovitz

sandra.iczkovitz@gmail.com

1 Centre for Prognosis Studies in the Rheumatic Diseases, Toronto Western Hospital, University of Toronto Lupus Clinic, EW, 1-412, 399 Bathurst Street, Toronto, ON M5T 2S8, Canada

2 GlaxoSmithKline Inc., Toronto, ON, Canada

3 MUHC Lupus and Vasculitis Clinic, McGill University, Montreal, QC, Canada

4 Centre de Rhumatologie, Quebec, Canada

5 McMaster University, Hamilton, ON, Canada

6 Dalhousie University, Halifax, Nova Scotia, Canada

7 Memorial University, St. John's, Newfoundland, Canada

8 Credit Valley Rheumatology, Canada, Mississauga, ON, Canada and $59.1 \%$ received a lower dose at Month 6. At Month $6,80.8 / 57.7 / 17.3 \%$ of patients had a physician-determined clinical improvement of $\geq 20 / \geq 50 / \geq 80 \%$, respectively. Sixteen patients had a SLE Disease Activity Index-2K score at both baseline and Month 6, with a mean improvement of $2.6 \pm 5.3$ from $8.1 \pm 3.2$ at baseline. No formal disease assessment tool was utilized for $42.3 \%$ of study patients at baseline. This study provides the first real-world insights into belimumab use in Canada. It demonstrates significant reduction or discontinuation of glucocorticoid dose in $70.5 \%$ of patients and clinically significant improvement following 6 months' belimumab therapy. The high number of patients with no formal disease activity assessments highlights a key care gap in SLE treatment in the real-world setting.

Keywords Lupus erythematosus, systemic $\cdot \mathrm{B}$-cell activating factor · Observational study $\cdot$ Glucocorticoids . Disease progression $\cdot$ Rheumatology

\section{Introduction/objectives}

Systemic lupus erythematosus (SLE) is a chronic autoimmune disease that affects approximately 31.9-51.0 per 100,000 persons in Canada, depending on the case definition used [1]. Standard therapies for SLE include glucocorticoids, antimalarial agents, non-steroidal antiinflammatory drugs (NSAIDs), cyclophosphamide, and immunosuppressive/immunomodulatory agents [2-6]. Despite improvements in medical care leading to improved survival of patients with SLE in recent decades [7], longterm morbidity can adversely affect patients' quality of life and their ability to work, resulting in substantial direct and indirect costs. 
Belimumab (Benlysta ${ }^{\mathrm{TM}}$ ) is a B-lymphocyte stimulator (BLyS)-specific inhibitor that blocks the binding of soluble BLyS, a B-cell survival factor, to its receptors on B cells [8]. Belimumab was approved in Canada in 2011 and is indicated in addition to standard therapy for reducing disease activity in adult patients with active, autoantibodypositive SLE [9]. Two pivotal Phase 3 randomized, doubleblind, placebo-controlled trials (BLISS-52 and BLISS-76), conducted in adults with SLE, demonstrated superiority of belimumab over placebo plus standard of care in the primary efficacy analysis of response rate, measured by the SLE responder index (SRI) at Week 52. Reductions in disease activity and severe flares were also observed [10, 11].

While the clinical efficacy of belimumab has been demonstrated in randomized controlled trials, utilization patterns and clinical benefit in real-world clinical practice have not been studied in Canada. The OBSErve studies (evaluation Of use of Belimumab in clinical practice SEttings) are observational studies designed to describe the overall patterns of SLE care and clinical outcomes following belimumab therapy in a real-world setting in different countries. The first of the OBSErve studies to report was the OBSErve US study (GSK study 117295), conducted in the USA, which investigated the clinical effectiveness, changes in laboratory parameters and healthcare resource utilization associated with belimumab plus standard of care in a cohort of patients with SLE over 24 months in clinical practice [12]. Here we present the results of the OBSErve Canada study (GSK Study 117300), which aimed to provide real-world insights on the characteristics of patients receiving belimumab in clinical practices in Canada, as well as to describe overall patterns of SLE care, clinical outcomes, including changes in glucocorticoid dose, following 6 months of therapy with belimumab in Canadian clinical practice settings.

\section{Methods}

\section{Study design}

This was a multicenter, retrospective, medical chart review study conducted in Canada. The study period included the 6-month treatment history period prior to the index date (belimumab initiation date; baseline) and a 6-month follow-up period after the index date.

Rheumatologists, who managed/treated $\geq 10$ patients with SLE, had $\geq 5$ years of experience in treating SLE and had $\geq 2$ patients currently receiving belimumab (of whom $\geq 1$ patient had received $\geq 8$ infusions) were recruited to participate in the study. Participating physicians were responsible for patient selection and enrollment, chart data abstraction and data validation. Data collection occurred by physician abstraction of patient medical chart data onto the paper-based physician practice profile form (PPPF) and study case report forms (CRFs). CRFs captured patient demographics, comorbidities, SLE disease characteristics including disease course and disease activity assessment, prior SLE treatments, belimumab initiation profile including reason for initiation, concomitant medications and healthcare resource use. All patient data were anonymized. While this study was not designed to collect data relating to safety of belimumab, nor was this an objective, available data pertaining to adverse events (AEs) were summarized and AEs that the physician deemed belimumab-related were reported to the study sponsor.

Central ethics approval for the study was obtained from the Veritas independent review board (IRB; project reference 'Evaluation of Use of Belimumab in Clinical Practice Settings [OBSErve]'). Institutional research ethics board approvals were also obtained from the University Health Network Research Ethics Board (147641-BE), Hamilton Integrated Research Ethics Board (13-743-C), and McGill University Health Centre Genetics/Population Research Investigator Initiated Studies Research Ethics Board (13-097 GEN). As patient data were anonymized, a waiver of informed consent for study patients was granted by the Veritas IRB.

\section{Patient population}

Physicians enrolled all patients from their practice who fulfilled the following inclusion criteria: adults aged $\geq 18$ years with a diagnosis of SLE based on the physician's judgement, who had received, as part of usual-care, $\geq 8$ belimumab infusions ( 6 months of continuous treatment); reason for belimumab initiation could be identified; $\geq 6$ months of medical history prior to belimumab initiation was documented with the participating study physician. Patients enrolled in any other SLE-related clinical trial were excluded.

\section{Primary study endpoints}

The primary study endpoints included: physiciandetermined SLE disease severity (mild, moderate, severe); patterns of SLE care among belimumab users in clinical practice in Canada; SLE clinical outcome measures at Month 6, as measured by physician-determined overall clinical status compared with baseline [worse, no improvement, and improvement $(<20, \geq 20, \geq 50, \geq 80)]$; and glucocorticoid use. 


\section{Other study endpoints}

Other study endpoints included measures of disease activity routinely used by physicians, such as the physician global assessment (PhGA), patient global assessment (PtGA), the Systemic Lupus Erythematosus Disease Activity Index 2000 (SLEDAI-2K) or its derivation, the British Isles Lupus Assessment Group (BILAG) score, and the Fatigue Severity Scale. The PtGA score is reported on a scale of 0 (no disease activity)-100 (high disease activity). Change in PtGA was calculated for patients who had a score recorded on the CRF at baseline and Month 6 .

\section{Statistical analyses}

Due to the largely descriptive nature of the study, no formal sample size calculations were undertaken to determine the number of physicians or patients to be included in the study. The study sample size was based solely on the number of physicians and patients who met the inclusion criteria at baseline. Descriptive statistics and univariate analyses were conducted where appropriate.

\section{Results}

\section{Physician characteristics}

Seven rheumatologists (four hospital-based, three community-based) were recruited. The majority $(n=6)$ practiced in urban settings. Four physicians were individual practitioners and three were members of a group practice. At the time of baseline data collection, physicians estimated that in total they were currently treating or managing a mean of $1814 \pm 684.2$ patients, of whom $176 \pm 113.7$ patients had SLE. Physicians reported a mean of $9 \pm 9.3$ patients currently receiving belimumab.

Four rheumatologists (57.1\%) reported routinely using the PhGA and the PtGA, and two physicians (28.6\%) each also reported routinely measuring SLEDAI-2K, BILAG score, and the Fatigue Severity Scale. Three rheumatologists $(42.9 \%)$ reported that they did not routinely use any disease activity instrument.

\section{Patient characteristics}

A total of 52 patients were included in the study. Patients were predominantly female $(94.2 \%)$ and largely Caucasian (65.4\%), followed by African origin (15.4\%), Asian (11.5\%), Hispanic (5.8\%), and West Indian (1.9\%). The mean (SD) age of the patients was 46.5 (10.8) years
(Table 1). Patients had a mean of $2.8 \pm 2.2$ comorbidities, the most common of which were hypertension (44.2\%), hyperlipidemia $(23.1 \%)$, depression $(15.4 \%)$, and fibromyalgia (13.5\%). Approximately $40 \%$ of patients were employed (34.6\% full time and 5.8\% part time) and 28.8, 13.5 , and $5.8 \%$ were reported as having disabled, unemployed, and retired employment status, respectively. The most frequently cited reasons for starting belimumab therapy were: previous treatment regimen was not effective $(69.2 \%)$; aim to decrease use or dose of glucocorticoids $(67.3 \%)$; worsening patient condition $(50.0 \%)$; and previous treatment regimen not well tolerated (17.3\%).

\section{SLE disease characteristics}

More than half of the patients $(53.8 \%)$ had been diagnosed with SLE for more than 10 years (Table 1). The most common clinical manifestations were musculoskeletal $(71.2 \%)$, mucocutaneous $(55.8 \%)$, immunologic $(30.8 \%)$, or constitutional (fatigue) $(28.8 \%)$. The most frequently reported specific SLE manifestations were arthritis (69.2\%), rashes (46.2\%), low complement (26.9\%), and increased anti-double-stranded DNA (antidsDNA) antibody levels (21.2\%). Other associated features frequently reported were fatigue $(28.8 \%)$ and alopecia $(21.2 \%)$. Physician-assessed SLE disease severity, at initial SLE diagnosis and at belimumab initiation, was reported as mild/moderate/severe in 5.8/51.9/42.3\% and $5.8 / 76.9 / 17.3 \%$ of patients, respectively. The majority (86.5\%) of patients had $\geq 2$ clinical SLE manifestations at belimumab initiation, with a mean of $3.1 \pm 1.5$ manifestations, ranging from 0.7 in patients with mild SLE to 3.2 in those with moderate or severe SLE.

In the 6 months prior to belimumab initiation, all patients received immunosuppressants, often in combination with oral glucocorticoids (96.2\%), antimalarials (92.3\%), and/or NSAIDs (55.8\%). Combination SLE therapy continued to be common at baseline, with most patients receiving oral glucocorticoids (84.6\%), antimalarials (76.9\%), and/or immunosuppressants $(73.1 \%)$ (Table 1). The most commonly prescribed immunosuppressants at baseline were mycophenolate mofetil (32.7\%), azathioprine (21.2\%), and methotrexate (17.3\%).

The most frequently administered disease activity assessments at belimumab initiation among the study patient population were the PtGA (48.1\%) and SLEDAI2K (or SELENA-SLEDAI) (32.7\%). The fatigue severity scale was available for $30.8 \%$ of patients, PhGA for $25.0 \%$, and the health assessment questionnaire for $25.0 \%$. Formal disease assessment instruments were not utilized for $42.3 \%$ (22/52) of study patients, both at baseline and Month 6. 
Table 1 Patient demographic and clinical characteristics

\begin{tabular}{|c|c|c|c|c|}
\hline & \multirow{2}{*}{$\begin{array}{l}\text { Total patients with } \\
\text { SLE }(n=52)\end{array}$} & \multicolumn{3}{|c|}{ SLE disease severity } \\
\hline & & Mild $(n=3)$ & Moderate $(n=40)$ & Severe $(n=9)$ \\
\hline Mean (SD) age (years) & $46.5(10.8)$ & $43.3(5.8)$ & $46.8(10.8)$ & $46.7(12.8)$ \\
\hline Gender: female, $n(\%)$ & $49(94.2)$ & $3(100.0)$ & $37(92.5)$ & $9(100.0)$ \\
\hline \multicolumn{5}{|l|}{ Race, $n(\%)$} \\
\hline Caucasian & $34(65.4)$ & $2(66.7)$ & $24(60.0)$ & $8(88.9)$ \\
\hline African origin & $8(15.4)$ & $1(33.3)$ & $7(17.5)$ & - \\
\hline Asian & $6(11.5)$ & - & $6(15.0)$ & - \\
\hline Hispanic & $3(5.8)$ & - & $3(7.5)$ & - \\
\hline West Indian & $1(1.9)$ & - & - & $1(11.1)$ \\
\hline \multicolumn{5}{|l|}{ Time since SLE diagnosis, $n(\%)$} \\
\hline $0-5$ years & $12(23.1)$ & - & $9(22.5)$ & $3(33.3)$ \\
\hline $6-10$ years & $12(23.1)$ & - & $10(25.0)$ & $2(22.2)$ \\
\hline$>10$ years & $28(53.8)$ & $3(100.0)$ & $21(52.5)$ & $4(44.4)$ \\
\hline \multicolumn{5}{|l|}{ SLE disease severity at baseline, $n(\%)$} \\
\hline Mild & $3(5.8)$ & $3(100.0)$ & - & - \\
\hline Moderate & $40(76.9)$ & - & $40(100.0)$ & - \\
\hline Severe & $9(17.3)$ & - & - & $9(100.0)$ \\
\hline \multicolumn{5}{|l|}{ SLE disease characteristics at baseline, $n(\%)$} \\
\hline High anti-dsDNA & $22(42.3)$ & $2(66.7)$ & $16(40.0)$ & $4(44.4)$ \\
\hline Low $\mathrm{C} 4$ & $20(38.5)$ & $2(66.7)$ & $15(37.5)$ & $3(33.3)$ \\
\hline Low C3 & $17(32.7)$ & $3(100.0)$ & $12(30.0)$ & $2(22.2)$ \\
\hline Leukopenia & $9(17.3)$ & - & $8(20.0)$ & $1(11.1)$ \\
\hline Proteinuria & $6(11.5)$ & - & $5(12.5)$ & $1(11.1)$ \\
\hline None & $16(30.8)$ & - & $12(30.0)$ & $4(44.4)$ \\
\hline \multicolumn{5}{|l|}{ Top 5 reasons for initiating belimumab, $n(\%)$} \\
\hline Previous treatment regimen ineffective & $36(69.2)$ & $2(66.7)$ & $27(67.5)$ & $7(77.8)$ \\
\hline Decrease use of glucocorticoids & $35(67.3)$ & $3(100.0)$ & $26(65.0)$ & $6(66.7)$ \\
\hline Worsening patient condition & $26(50.0)$ & $1(33.3)$ & $18(45.0)$ & $7(77.8)$ \\
\hline Previous treatment regimen not well tolerated & $9(17.3)$ & - & $8(20.0)$ & $1(11.1)$ \\
\hline Patient request & $2(3.8)$ & - & $2(5.0)$ & - \\
\hline \multicolumn{5}{|l|}{ Concomitant SLE medications, $n(\%)$} \\
\hline Oral glucocorticoids & $44(84.6)$ & $3(100.0)$ & $32(80.0)$ & $9(100.0)$ \\
\hline Antimalarials & $40(76.9)$ & $3(100.0)$ & $32(80.0)$ & $5(55.6)$ \\
\hline Immunosuppressant $^{\mathrm{a}}$ & $38(73.1)$ & $2(66.7)$ & $30(75.0)$ & $6(66.7)$ \\
\hline NSAIDs & $8(15.4)$ & - & $6(15.0)$ & $2(22.0)$ \\
\hline Mean (SD) prednisone equivalent dose (mg/day) & $13.6(10.0)$ & $13.3(14.4)$ & $13.3(9.8)$ & $14.7(10.5)$ \\
\hline $\begin{array}{l}\text { Mean (SD) prednisone equivalent dose in patients with } \\
\geq 7.5 \mathrm{mg} / \text { day at index date }(\mathrm{mg} / \text { day })\end{array}$ & $17.8(9.5)$ & - & - & - \\
\hline
\end{tabular}

Anti-dsDNA anti-double-stranded DNA, $C$ complement, NSAID non-steroidal anti-inflammatory drug, $S D$ standard deviation, $S L E$ systemic lupus erythematosus

${ }^{a}$ Immunosuppressants included mycophenolate mofetil, azathioprine, methotrexate, cyclosporine, and mycophenolate sodium 


\section{Clinical outcome measures}

\section{Overall clinical response}

Following 6 months of belimumab treatment, 80.8, 57.7, and $17.3 \%$ of patients were observed by their physicians to have an overall clinical improvement of $\geq 20, \geq 50$, and $\geq 80 \%$, respectively (Table 2 ). Nine patients $(17.3 \%$ ) had $<20 \%$ clinical improvement and one patient (1.9\%) showed no improvement; no patients were reported to have had worsened disease. Similar trends in improvement were observed across the disease severity subgroups (Table 2 ).

\section{Glucocorticoid use}

Of the 44 patients receiving oral glucocorticoids at baseline, five (11.4\%) discontinued oral glucocorticoid therapy after 6 months of belimumab therapy (Table 3). Among patients with SLE who were still receiving oral glucocorticoids after 6 months of belimumab therapy, the glucocorticoid dose was decreased for $59.1 \%$, remained the same for $22.7 \%$, and was increased for $6.8 \%$ of patients (Table 3). At Month 6, the mean glucocorticoid dose had decreased by $5.8 \mathrm{mg} /$ day from baseline (Table 4). The mean reduction among patients with a baseline glucocorticoid dose $\geq 7.5 \mathrm{mg} /$ day was $8.2 \pm 9.8 \mathrm{mg} /$ day after 6 months of belimumab therapy (dose at belimumab initiation $17.8 \pm 9.5 \mathrm{mg} /$ day; dose following 6 months of belimumab $9.6 \pm 6.0 \mathrm{mg} /$ day).

\section{Other concomitant medications}

The percentage of patients receiving antimalarials at Month 6 remained similar to baseline ( 78.8 vs. $76.9 \%$ ), as did the percentage of patients receiving immunosuppressants (73.1 vs. $69.2 \%$ ). The percentage receiving NSAIDs remained the same $(15.4 \%)$.
Table 2 Physician-determined clinical improvement from baseline at Month 6

\begin{tabular}{lclll}
\hline & All patients $(n=52)$ & Mild SLE $(n=3)$ & $\begin{array}{l}\text { Moderate } \\
\text { SLE }(n=40)\end{array}$ & Severe SLE $(n=9)$ \\
\hline Worsened, $n(\%)$ & $0(0)$ & $0(0)$ & $0(0)$ & $0(0)$ \\
No improvement, $n(\%)$ & $1(1.9)$ & $0(0)$ & $1(2.5)$ & $0(0)$ \\
$<20 \%$ improvement, $n(\%)$ & $9(17.3)$ & $0(0)$ & $7(17.5)$ & $2(22.2)$ \\
$\geq 20 \%$ improvement, $n(\%)$ & $42(80.8)$ & $3(100.0)$ & $32(80.0)$ & $7(77.8)$ \\
$\geq 50 \%$ improvement, $n(\%)$ & $30(57.7)$ & $3(100.0)$ & $23(57.5)$ & $4(44.4)$ \\
$\geq 80 \%$ improvement, $n(\%)$ & $9(17.3)$ & $2(66.7)$ & $5(12.5)$ & $2(22.2)$ \\
\hline
\end{tabular}

SLE systemic lupus erythematosus

\begin{tabular}{lclcl}
\hline & All patients $(n=44)$ & Mild SLE $(n=3)$ & $\begin{array}{l}\text { Moderate SLE } \\
(n=32)\end{array}$ & Severe SLE $(n=9)$ \\
\hline Discontinued, $n(\%)$ & $5(11.4)$ & $1(33.3)$ & $4(12.5)$ & $0(0)$ \\
Decreased, $n(\%)$ & $26(59.1)$ & $0(0)$ & $20(62.5)$ & $6(66.7)$ \\
No change, $n(\%)$ & $10(22.7)$ & $2(66.7)$ & $7(21.9)$ & $1(11.1)$ \\
Increased, $n(\%)$ & $3(6.8)$ & $0(0)$ & $1(3.1)$ & $2(22.2)$ \\
\hline
\end{tabular}

$S L E$ systemic lupus erythematosus
Table 3 Change in glucocorticoid use from baseline to Month 6 


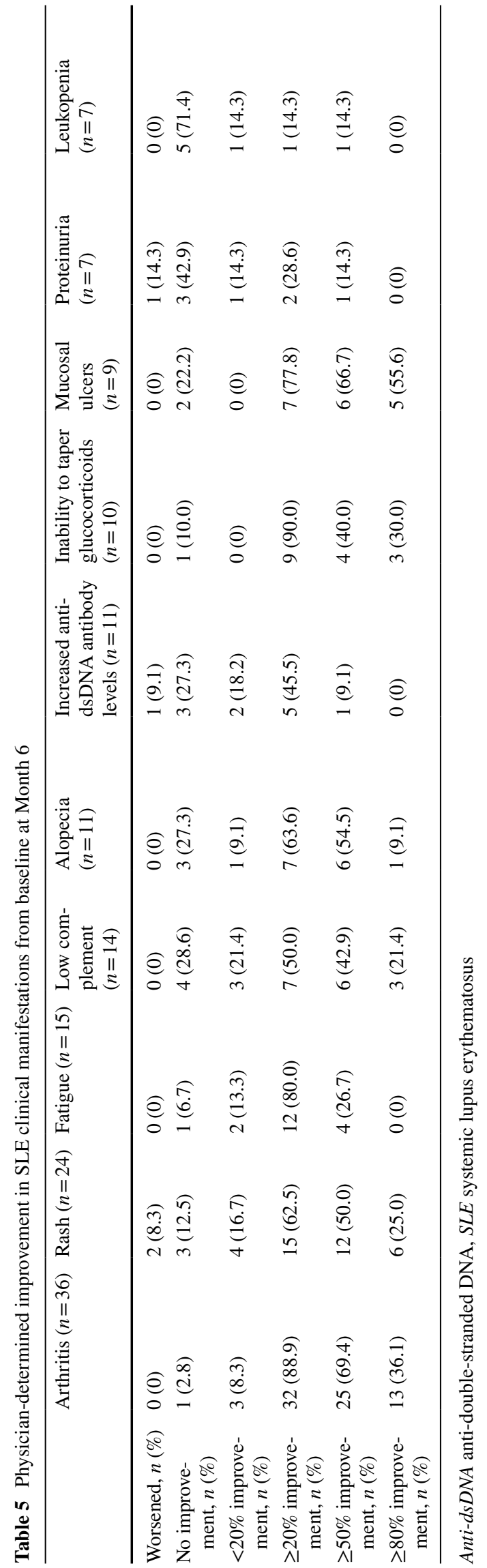

\section{SLE manifestations}

In patients reported to have arthritis manifestations at baseline ( $n=36$ ), a clinical response of $\geq 20, \geq 50$, and $\geq 80 \%$ improvement in SLE disease severity following 6 months of belimumab therapy was reported in 88.9, 69.4, and $36.1 \%$ of patients, respectively. Improvements in other SLE clinical manifestations were also observed from baseline to Month 6 (Table 5); however, it is important to note that patient numbers were low in some groups.

\section{SLE disease activity assessment}

Among patients with assessments at both baseline and Month 6, mean PhGA score improved by $18.4(n=11)$ and mean SLEDAI-2K score improved by $2.6 \quad(n=16)$ (Table 6). Among patients with assessments at both baseline and Month $6(n=22)$, mean PtGA score improved by 0.5 over 6 months.

\section{Healthcare resource utilization}

Over the 6 months prior to belimumab initiation, two patients $(3.8 \%)$ were hospitalized a mean of 2.5 times, for a mean duration of 8.0 days; one patient had moderate SLE and the other had severe SLE. Only one (1.9\%) patient visited the emergency room in the 6 months prior to belimumab initiation. Hospitalizations remained infrequent during the belimumab treatment period: one patient with severe SLE had five admissions to hospital during the first 6 months on belimumab, with a total duration of 49 days. Six (11.5\%) patients had an unscheduled visit to their rheumatologist, likely for disease management, during the 6 months prior to belimumab initiation; incidence of unscheduled visits was similar during belimumab treatment [5 (9.6\%) patients].

\section{Adverse events}

AEs were reported in 12 patients (17 AEs in total); those that occurred in $\geq 1$ patient were sinusitis/sinus infection $(n=3)$, diarrhea $(n=2)$, and cephalalgia $(n=2)$.

\section{Discussion}

This was a non-interventional, observational retrospective medical chart review study executed in Canada. This study was commissioned to examine adult patients with SLE, receiving belimumab, in real-world clinical practice settings. The primary objective was to describe overall patterns of SLE care in adult patients with SLE prescribed $\geq 8$ infusions of belimumab over a 6-month 
Table 6 SLE disease activity scores at baseline and Month 6

\begin{tabular}{|c|c|c|c|c|}
\hline & \multirow{2}{*}{$\begin{array}{l}\text { Total patients with } \\
\text { SLE }(n=52)\end{array}$} & \multicolumn{3}{|c|}{ SLE disease severity at baseline } \\
\hline & & Mild $(n=3)$ & Moderate $(n=40)$ & Severe $(n=9)$ \\
\hline \multicolumn{5}{|l|}{ PtGA } \\
\hline Total patients with score at baseline and Month $6(n)$ & 22 & 3 & 15 & 4 \\
\hline Mean (SD) score at baseline & $60.5(22.6)$ & $46.7(10.1)$ & $61.4(23.0)$ & $67.3(28.2)$ \\
\hline Mean (SD) improvement from baseline to Month 6 & $0.5(24.0)$ & $0.7(17.1)$ & $2.6(26.6)$ & $-7.5(20.4)$ \\
\hline \multicolumn{5}{|l|}{$\mathrm{PhGA}$} \\
\hline Total patients with score at baseline and Month $6(n)$ & 11 & 3 & 7 & 1 \\
\hline Mean (SD) score at baseline & $39.1(24.4)$ & $10.3(17.9)$ & $49.0(17.6)$ & $56.0(0.0)$ \\
\hline Mean (SD) improvement from baseline to Month 6 & $18.4(15.9)$ & $8.3(14.4)$ & $26.3(11.0)$ & $-7.0(0.0)$ \\
\hline \multicolumn{5}{|l|}{ SLEDAI- $2 \mathrm{~K}^{\mathrm{a}}$} \\
\hline Total patients with score at baseline and Month $6(n)$ & 16 & 3 & 10 & 3 \\
\hline Mean (SD) score at baseline & $8.1(3.2)$ & $4.0(0.0)$ & $8.4(2.8)$ & $11.3(1.2)$ \\
\hline Mean (SD) improvement from baseline to Month 6 & $2.6(5.3)$ & $0.0(0.0)$ & $3.0(3.3)$ & $4.0(12.2)$ \\
\hline
\end{tabular}

PhGA physician global assessment, PtGA patient global assessment, SD standard deviation, SELENA-SLEDAI Safety of Estrogens in Lupus Erythematosus National Assessment-Systemic Lupus Erythematosus Disease Activity Index, SLE systemic lupus erythematosus

${ }^{a}$ SLEDAI-2K and its derivations including SELENA-SLEDAI

period, and to examine clinical status and outcomes, including glucocorticoid use.

The patient population in this real-world setting were similar to those seen in the belimumab clinical trial program in terms of manifestations, with musculoskeletal, mucocutaneous, and immunology being the most common clinical manifestations $[10,11]$. In this real-world population, $42.3 \%$ of patients had elevated anti-dsDNA antibody levels at baseline. In the BLISS-52 and BLISS76 trials, patients were required to be positive for antinuclear antibodies (ANA) or anti-dsDNA to participate. The proportions of patients with anti-dsDNA $\geq 30 \mathrm{IU} / \mathrm{ml}$ were slightly higher in these trials $(63-77 \%$ across treatment groups) compared with the population in the present study $[10,11]$.

Prolonged use of high-dose glucocorticoids in SLE can cause long-term organ damage and morbidity and therefore, glucocorticoid reduction is an important factor in treatment of SLE [13]. In this study, we observed a decrease in glucocorticoid use following 6 months of belimumab therapy, with $59.4 \%$ of patients reducing their daily glucocorticoid dose and $11.4 \%$ of patients discontinuing glucocorticoid use. It should also be noted that the proportion of patients receiving glucocorticoids decreased from 96.2 to $84.6 \%$ between 6 months prior to baseline and baseline. Reductions in glucocorticoid dosage following 6 months of therapy with belimumab were more pronounced in the high glucocorticoid group (>7.5 mg/day; $-8.2 \mathrm{mg} /$ day) compared with all patients $(-5.8 \mathrm{mg} / \mathrm{day})$. Similar results were seen in the OBSErve US study, in which $9 \%$ of patients discontinued glucocorticoids and $77 \%$ received a lower dose at Month 6. In the OBSErve US study, the percentage of patients prescribed glucocorticoids, and the dose received, continued to decrease from Month 6 to Month 24 [12].

Regular assessment of disease activity in SLE is vital for effective treatment management, as well as to prevent the long-term consequences of persistent disease activity (e.g., organ damage, mortality, high-dose glucocorticoid use). Although both the American College of Rheumatology and the European League Against Rheumatism guidelines recommend regular monitoring of disease activity in patients with SLE $[14,15]$, there is no standard disease assessment tool for SLE recommended by rheumatology professional organizations. The present study found that the clinical assessment instruments most frequently used by physicians treating patients receiving belimumab in Canada were the PtGA and PhGA [used by 4/7 (57.1\%) physicians each] and SLEDAI-2K, BILAG, and patient-reported outcomes on fatigue using the Fatigue Severity Scale [used by 2/7 (28.6\%) physicians each]. Consistent with the findings of OBSErve US, which showed that $50 \%$ of physicians did not use any disease assessment tool [13], in this study we found that no formal disease assessment tool was utilized for $42.3 \%$ of study patients. This highlights a key care gap in the treatment of patients with SLE.

It is imperative that disease activity be measured, ideally with standardized and validated tools, prior to initiation of SLE treatments to adequately assess disease progression and patient response. The lack of use of current disease activity assessment instruments suggests an urgent need for Canadian guidelines on the assessment of patients with lupus. Rheumatologists managing other rheumatic diseases, such as rheumatoid arthritis and psoriatic arthritis, utilize standardized and validated disease activity tools 
(physician-derived and patient-reported outcomes) when submitting applications for biologic treatment approval and renewal. Consistent with these other biologics, many medical insurance companies require patients with SLE to have SLEDAI-2K $\geq 6$ with positive serology (e.g., ANA, anti-dsDNA antibodies, anti-Smith antibodies, low complement) to qualify for treatment with belimumab. This could suggest that rheumatologists managing patients with lupus may be required in the future to use validated instruments for the management of patients in their clinic.

Given the absence of a standard disease assessment tool for SLE recommended by rheumatology professional organizations, the overall clinical response improvement was physician-assessed. We observed that treatment for 6 months with belimumab led to clinically significant improvements, as measured by physicians' overall assessment, and the reduction or discontinuation of glucocorticoids. Over $80 \%$ of patients experienced a $\geq 20 \%$ improvement in physician-assessed disease severity and almost $60 \%$ experienced $\mathrm{a} \geq 50 \%$ improvement in physician-assessed disease severity. Less than $2 \%$ of patients experienced no change in symptoms and no patients were reported by their physicians to have worsened. These results are comparable to those from the OBSErve US study, in which 88.4 and $48.7 \%$ of patients achieved $\geq 20$ and $\geq 50 \%$ improvement in overall clinical response following 6 months of belimumab therapy [12]. Hospitalizations and unscheduled visits to a rheumatologist were similar and infrequent both prior to, and during, belimumab treatment.

Observational studies enable data collection from a population of patients that provide a more realistic reflection of real-world clinical practice vs. the strictly controlled patient populations included in clinical trials. In this retrospective chart review study, a major limitation was that the quantification of clinical response (primary outcome) was a non-standardized, subjective tool that has not been validated. The lack of a control group and no randomization to treatment risks bias in efficacy measurement. Nevertheless, the data show that $>70 \%$ of patients were able to decrease or stop glucocorticoids, which is a quantitative endpoint, suggesting the effectiveness and tolerability of belimumab. However, the primary aim of this study was not to determine efficacy, but to establish real-world insights into the use of belimumab in clinical practice.

The requirement of at least 6 months of treatment (for 8 doses) strongly biases this study population in favor of those who responded to and tolerated belimumab treatment, or were treated by physicians who were compliant with regular patient follow-up. This may limit comparisons with other studies of belimumab use in real-world patients with SLE. Furthermore, the majority of the patients were Caucasian (65.4\%), followed by of African origin (15.4\%) and this may limit the generalizability of our results. Due to the number of physicians and patients participating in this study and the availability of data, some of the analyses were conducted with low patient numbers (e.g., the physician-determined improvement in SLE clinical manifestations from baseline to 6 months) and therefore should be interpreted with caution. Other limitations include that patients were included in the study based on the physician's judgment of a diagnosis of SLE, and that information collected on the patient CRF and PPPF included only best estimates of the current SLE-treated patients and subgroups and did not equate with a complete, detailed examination and analysis of all actual records for patients with SLE at each physician's site/practice. In addition, the CRFs are an interpretation of what was written on the patient charts and under-reporting may have occurred if not all events were recorded on the patient charts. Physicians had varying levels of experience with validated assessment instruments for SLE disease activity, and causal inference of any effect of belimumab cannot be made as the study population did not include a control arm. In this study, the majority of the patients were Caucasian $(65.4 \%)$ followed by African origin $(15.4 \%)$ and therefore this imposes limitation on the generalizability of our results.

This uncontrolled retrospective chart review supports the results of previous clinical trials in a real-world setting. Key findings included the reduction or discontinuation of glucocorticoids, which has important clinical implications given the adverse effects of long-term use of this therapy, and the suggestion of clinically significant improvement as measured by physicians' overall assessment and SLEDAI$2 \mathrm{~K}$ scores. The high number of patients with no formal disease activity assessments highlights a key care gap in the treatment of SLE in the real-world setting.

Acknowledgements The authors thank Medical Data Analytics, who conducted the study on behalf of GSK Canada. This study was funded by GSK. Medical writing support was provided by Louisa Pettinger, PhD, of Fishawack Indicia Ltd funded by GSK.

\section{Compliance with ethical standards}

Central ethics approval for the study was obtained from the Veritas independent review board [IRB; project reference 'Evaluation of Use of Belimumab in Clinical Practice Settings (OBSErve)']. Institutional research ethics board approvals were also obtained from the University Health Network Research Ethics Board (14-7641-BE), Hamilton Integrated Research Ethics Board (13-743-C), and McGill University Health Centre Genetics/Population Research Investigator Initiated Studies Research Ethics Board (13-097 GEN). As patient data were anonymized, a waiver of informed consent for study patients was granted by the Veritas IRB.

\section{Sources of support Study 117300 was funded by GSK.}

Conflict of interest Amyn Sayani is an employee of and holds stocks in GSK. Zahi Touma is a consultant to GSK, EMD Serono Research and Development, Biogen Idec, and Janssen. Mark Matsos is a consult- 
ant to Genzyme, AbbVie, Janssen, Bristol-Myers Squibb, and UCB. Christian A. Pineau has received research support from GSK. George A. Ecker has no financial disclosures. Andrew Chow has received clinical trials support from AbbVie, Bristol-Myers Squibb, Celgene, GSK, Janssen, Pfizer, UCB and is a speaker/consultant for AbbVie, Bristol-Myers Squibb, Celgene, Janssen, Novartis, Pfizer, Takeda, UCB. Isabelle Fortin has received research support from AbbVie, Amgen, Bristol-Myers Squibb, Pfizer, AstraZeneca, and UCB. Sandra Iczkovitz was an employee of GSK at the time of the study.

Open Access This article is distributed under the terms of the Creative Commons Attribution 4.0 International License (http:// creativecommons.org/licenses/by/4.0/), which permits unrestricted use, distribution, and reproduction in any medium, provided you give appropriate credit to the original author(s) and the source, provide a link to the Creative Commons license, and indicate if changes were made.

\section{References}

1. Bernatsky S, Joseph L, Pineau CA, Tamblyn R, Feldman DE, Clarke AE (2007) A population-based assessment of systemic lupus erythematosus incidence and prevalence-results and implications of using administrative data for epidemiological studies. Rheumatology 46:1814-1818

2. Chatham WW, Kimberly RP (2001) Treatment of lupus with corticosteroids. Lupus 10:140-147

3. Ginzler EM, Dooley MA, Aranow C, Kim MY, Buyon J, Merrill JT et al (2005) Mycophenolate mofetil or intravenous cyclophosphamide for lupus nephritis. N Engl J Med 353:2219-2228

4. Houssiau FA, Vasconcelos C, D'Cruz D, Sebastiani GD, Garrido Ed Ede R, Danieli MG et al (2002) Immunosuppressive therapy in lupus nephritis: the Euro-Lupus Nephritis Trial, a randomized trial of low-dose versus high-dose intravenous cyclophosphamide. Arthritis Rheum 46:2121-2131

5. Ruiz-Irastorza G, Khamashta MA, Castellino G, Hughes GR (2001) Systemic lupus erythematosus. Lancet 357:1027-1032

6. Wallace DJ (2002) Management of lupus erythematosus: recent insights. Curr Opin Rheumatol 14:212-219
7. Trager J, Ward MM (2001) Mortality and causes of death in systemic lupus erythematosus. Curr Opin Rheumatol 13:345-351

8. Baker KP, Edwards BM, Main SH, Choi GH, Wager RE, Halpern WG et al (2003) Generation and characterization of LymphoStat-B, a human monoclonal antibody that antagonizes the bioactivities of B lymphocyte stimulator. Arthritis Rheum 48:3253-3265

9. Health Canada (2011) Summary basis of decision BENLYSTA, belimumab $120 \mathrm{mg} / \mathrm{vial}$ and $400 \mathrm{mg} / \mathrm{vial}$, powder for solution, GlaxoSmithKline Inc., Submission Control Number 137699, Ontario

10. Furie R, Petri M, Zamani O, Cervera R, Wallace DJ, Tegzova D et al (2011) A phase III, randomized, placebo-controlled study of belimumab, a monoclonal antibody that inhibits B lymphocyte stimulator, in patients with systemic lupus erythematosus. Arthritis Rheum 63:3918-3930

11. Navarra SV, Guzman RM, Gallacher AE, Hall S, Levy RA, Jimenez RE et al (2011) Efficacy and safety of belimumab in patients with active systemic lupus erythematosus: a randomised, placebo-controlled, phase 3 trial. Lancet 377:72-731

12. Collins CE, Dall'Era M, Kan H, Macahilig C, Molta C, Koscielny V et al (2016) Response to belimumab among patients with systemic lupus erythematosus in clinical practice settings: 24-month results from the OBSErve study in the USA. Lupus Sci Med 3:e000118

13. Zonana-Nacach A, Barr SG, Magder LS, Petri M (2000) Damage in systemic lupus erythematosus and its association with corticosteroids. Arthritis Rheum 43:1801-1808

14. American College of Rheumatology (1999) Guidelines for referral and management of systemic lupus erythematosus in adults. American college of rheumatology ad hoc committee on systemic lupus erythematosus guidelines. Arthritis Rheum 42:1785-1796

15. Mosca M, Tani C, Aringer M, Bombardieri S, Boumpas D, Brey $\mathrm{R}$ et al (2010) European League Against Rheumatism recommendations for monitoring patients with systemic lupus erythematosus in clinical practice and in observational studies. Ann Rheum Dis 69:1269-1274 